\title{
A SURVEY ON FULL REFERENCE IMAGE QUALITY ASSESSMENT ALGORITHMS
}

\author{
Alphy George ${ }^{1}$, S. John Livingston ${ }^{2}$ \\ ${ }^{1}$ PG Student, Computer Science and Engineering, Karunya University, Tamilnadu, India, alphygeorge91@gmail.com \\ ${ }^{2}$ Asst. Professor, School of Computer Science, Karunya University, Tamilnadu, India, johnlivingston@karunya.edu
}

\begin{abstract}
Image quality measurement is very important for various image processing applications such as recognition, retrieval, classification, compression, restoration and similar fields. The images may contain different types of distortions like blur, noise, contrast change etc. So it is essential to rate the image quality appropriately. Traditionally subjective rating methods are used to evaluate the quality of the image, in which humans rated the image quality based on time requirements. This is a costly process and it needs experts for evaluating image quality. Nowadays many image quality assessment algorithms are available for finding the quality of images. These are mainly based on the properties of human visual system. These image quality assessment algorithms are the objective methods for finding quality. Most of the methods are heuristic and limited to specific application environment. Whereas some methods perform efficiently and having comparable performance with the subjective ratings. Objective methods are easy for integrating into various image compression techniques and other image processing applications. Based on the availability of the image, image quality assessment algorithms are classified into full reference, reduced reference and no reference respectively. Full reference algorithms normally adopt a two stage structure including local quality measurement and pooling to get the quality value. The input for this two stage structure includes a reference image and a distorted image. This paper presents a survey on the existing image quality assessment algorithms based on full reference method, in which a reference image will be available for finding the quality of the distorted image.
\end{abstract}

Keywords: Image distortion, Image quality assessment, Human visual system

\section{INTRODUCTION}

Image distortion is often present in almost all images. Different types of distortion are there. For example noise, blur, contrast change etc. These distortions can degrade the entire quality of the image. For example in image compression, if the captured image contains distortions then it would not match with the original image that is stored in the database. So finding the quality of the image in those areas is very necessary.

Traditionally subjective rating methods are used for measuring the quality of the image. In this subjective rating, humans rated the image quality. The images are given to the experts. Based on the time requirements available, they give ratings to the image. Subjective results can provide accurate results, but it is time consuming and also a costly process [11]. This leads to the development of objective image quality assessment algorithms (IQA) that will predict the quality of the image automatically. According to the availability of the original reference image, the objective methods are classified into full reference, reduced reference and no reference [6]. In full reference method a complete distortion free image is available for the comparison. In case of no reference method blind prediction of the image quality will be done. In the third method, only a portion of the reference image is available in the form of some extracted features. That's why that method is known as reduced reference method.

Most of the IQA metrics are based on the full reference method. Traditional predictors for the image quality are MSE (Mean Squared Error) and PSNR (Peak Signal-to Noise Ratio). MSE is a simple index based metric for measuring quality. But these are the poor predictors of image quality. In case of correlation with the human visual perception of quality, MSE shows poor performance [2]. Recently many methods have been developed in the area of image quality measurement based on the properties of human visual system (HVS). For the full reference methods, commonly a two stage structure is adopted. This two stage structure of full reference image quality assessment [8] is given in fig. 1.

These image quality measures can be implemented in various image compression algorithms and other image processing applications. In this way we can find if there is any distortions present in the image. This can be concluded if there is any degradation in the image quality by evaluating the quality metric value. On the basis of this information, we can remove that distorted image and replace with another good one. For evaluating the performance of IQA algorithms, many 
databases are publicly available. Some of the image distortions that are present in images taken from csiq database are shown in fig. 2 [10].

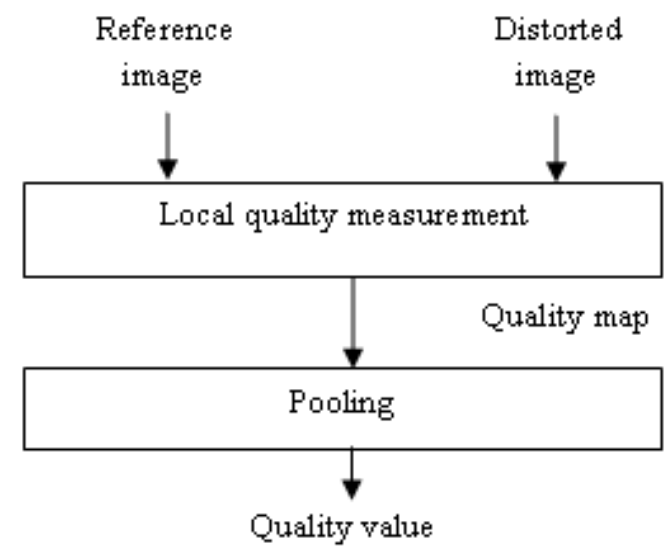

Fig -1: Full reference image quality assessment calculation

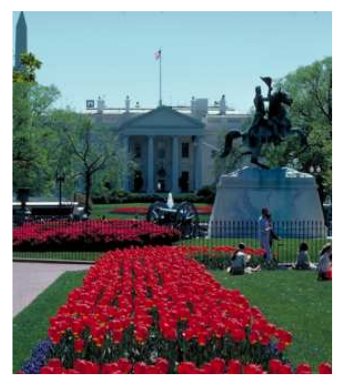

(a)

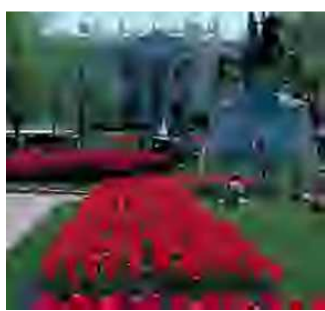

(c)

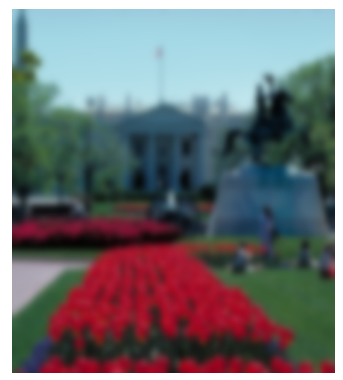

(b)

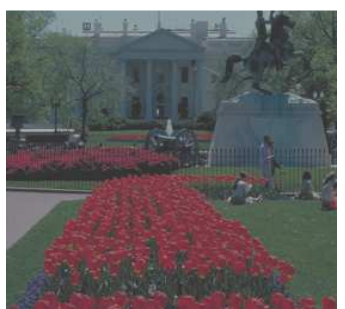

(d)
Fig -2: Images taken from the CSIQ database (a) original image (b) blurring (c) JPEG 2000 (d) contrast change

\section{FULL REFERENCE IMAGE QUALITY ASSESSMENT ALGORITHMS}

There are many methods available for image quality evaluation proposed by many researchers. This survey concentrates on various algorithms for the image quality assessment metrics. Various performance metrics are used to compare the image quality algorithms. This is also mentioned in this paper.

\subsection{SSIM (Structural Similarity Index Measure)}

This algorithm [6] is based on the concept that human visual system is highly adapted for extracting structural information from an image. So from the available image information in the original and distorted image, a quality measure is constructed. For getting the structural information, the influence of illumination has to be separated from the image. For that first calculate the luminance information of the two image signals $x$ and $\mathrm{y}$ in the form of mean intensity $\mu_{x}$ and $\mu_{y}$. The luminance comparison function $l(x, y)$ is then the comparison of $\mu_{x}$ and $\mu_{y}$. Then remove this mean intensity from the image signal. The resultant signal is in the form $x-\mu_{x}$. In the second step signal contrast is found using the standard deviation $\sigma_{x}$ and $\sigma_{y}$. The contrast comparison $c(x, y)$ is then the comparison of $\sigma_{x}$ and $\sigma_{y}$. In the third step the two image signals are divided by its own standard deviation. This will give the resultant signals $\left(x-\mu_{x}\right) / \sigma_{x}$ and $\left(x-\mu_{y}\right) / \sigma_{y}$. On these signals the structure comparison $s(x, y)$ is performed. In the final step the three components have to be combined. This will give the resultant similarity measure $S(x, y)$ i.e., $S(x, y)=f(l(x, y), c(x, y), s(x, y)$. This image quality assessment approach is applied using a sliding window algorithm. The window moves across the entire image. For each window the SSIM metric is measured.

\subsection{MAD (Most Apparent Distortion)}

Most apparent distortion [3] is mainly based on the property of HVS when judging the image quality. It consists of two strategies for quantifying the information contained in images. Detection based strategy and Appearance based strategy. In detection based strategy there are two steps that have to be done. First find the locations in which distortions are visible. This can be done by converting the original and the distorted images into luminance images and then converting it into perceived luminance. Applying a contrast sensitivity function to this will give the visibility map. In the second step combine this visibility map with the local errors. This detection based strategy mainly used for high quality images. For low quality images detection based strategy can be used. A log-Gabor decomposition method can be adopted for decomposing the original image into different subbands. Comparing the computed local subband statistics would give the result of appearance based strategy. The final prediction can get by combining the two strategies. 


\subsection{NPID (Normalized Perceptual Information}

\section{Distance)}

This image distortion analysis method [9] is mainly based on the theory of kolmogorov complexity which is rarely been studied in the field of image processing. Another important theory used here is the normalized information distance. Normalized information formula is calculated using the kolmogorov complexity. These two concepts are theoretical concepts and practically non computable [12]. This image quality measure is wavelet based in which the original and the distorted images are decomposed into different subbands using a laplacian pyramid subband decomposition method. Assuming the local independence and decorrelation among the subbands, kolmogorov complexity is approximated as

$$
K(x \mid y)=\sum_{i=1}^{n} K\left(x_{n} \mid y_{n}\right)
$$

Where $x_{n}$ and $y_{n}$ are wavelet subbands of the original and the distorted image respectively and $K$ is the kolmogorov complexity of the image. Because of the non computable nature of kolmogorov complexity, that can be approximated using the Shannon entropy. Because of the varying nature of subband coefficients, it is approximated using information content. The mutual information that is shared between the original and distorted image subbands is taken for the metric calculation. A gaussian scale mixture model (GSM) [14] is adopted for modeling the subband coefficients of the image and for the noise distortions in the HVS a gaussian channel model is adopted. Using these models, the final quality metric is derived. Finally, an information content weighting method is used to get more accurate value for the quality.

\subsection{MS-SSIM (Multi Scale Structural Similarity}

\section{Index Measure)}

The multi scale structural similarity measure [7] is a more flexible method than the other single scale methods. The existing SSIM algorithm is a single scale approach. By using this multi scale method image details with different resolutions can be incorporated. Low-pass filtering and down sampling are the two main operations used in this method. The original and the distorted images are iteratively low-pass filtered and then down sampling will be done on that by a factor of 2. For this multi scale operation, the original image is taken as scale 1 . The highest scale is scale $M$, so a total of $M-1$ iterations are taken place. Similar to the SSIM method, three comparisons have been done here i.e., contrast comparison, luminance comparison and the structure comparison. Only luminance comparison is performed on scale $M$. Other two are performed on the intermediate scales. The final quality measure is the combination of these three comparisons. This is a convenient image quality metric than the other single scale approaches.

\subsection{FSIM (Feature Based Structural Similarity Index)}

This feature based similarity index [5] is mainly based on the low level features of an image. Most of the methods are based on structural similarity. Two features are used here for the calculation of similarity index, phase congruency (PC) and gradient magnitude (GM) where $\mathrm{PC}$ is the primary feature and GM is the secondary feature. PC is a dimensionless measure. For the computation of FSIM, PC and GM have to be extracted from the image. The PC extraction can be done by using a log Gabor filter. The gradient operators can be Sobel, Prewitt and Schar operator. The input images can be gray scale or color images. If it is a color image, PC and GM can be extracted from the luminance. One of the main advantage of FSIM is that it can support color images. Sequence of steps involved in FSIM calculation is given in Fig. 3.

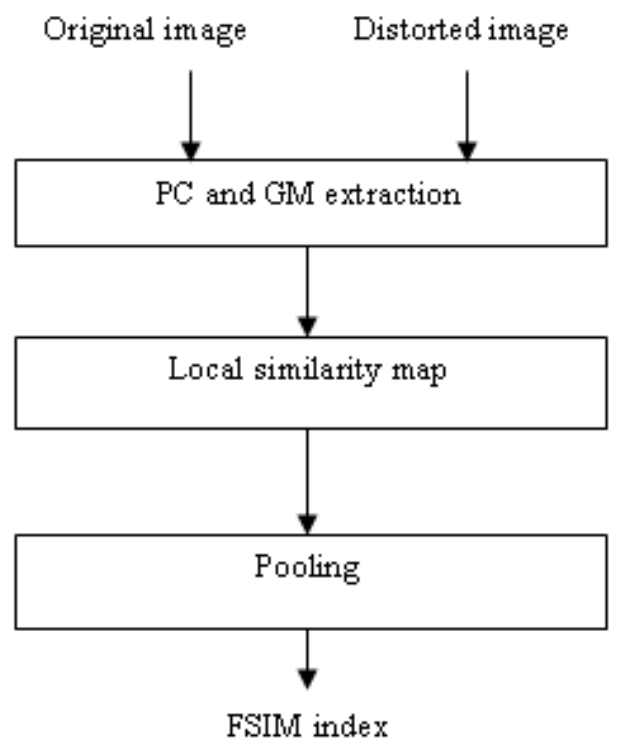

Fig -3: FSIM calculation

\subsection{Practical Image Quality Index}

This full reference quality metric [1] is mainly based on the CSF (Contrast Sensitivity Function). It is having a comparable performance advantages with the other existing image quality measurement algorithms. This algorithm is also based on the texture masking effect. This is a wavelet based method in which the image is divided into different subbands using a wavelet decomposition method. It is based on the assumption that the local distortion and the subband distortion contribute the entire distortion of the image. Already existing contrast sensitivity function (CSF) [13] is extended here and creating a new metric known as DCTex. CSF is used to model spatial frequency transforms. The proposed DCTex metric is the combination of contrast sensitivity function and the texture 
masking effect. Global smoothness and local brightness are the two masking parameters used in the metric. For the metric calculation, a sliding window is used that divides the original image and the distorted image into $8 \times 8$ non overlapping blocks. This proposed metric can be easily integrated into the JPEG compression standard.

\subsection{Content Partitioned Structural Similarity Index}

The content partitioned similarity index [4] is a modified version of SSIM and MS-SSIM. It is a four component image quality metric in which the original and the distorted images are divided into four component regions. The four regions are smooth regions, changed edges, preserved edges and texture regions. If the estimation of gradient magnitude is large then the two edges that is, preserved edges and changed edges can be found. If the magnitude is small, then smooth regions can be found. Between the low estimation and large estimation, texture regions can be found. The SSIM index has to be calculated for finding the image quality. A weighting method is adopted for the SSIM values. The values at the edge region are treated by giving larger weights because edges are having more importance in images. For the final calculation of the metric, a pooling method is adopted so that the average of the weighted values can be taken. The different steps involved in the metric calculation are given in Fig. 4.

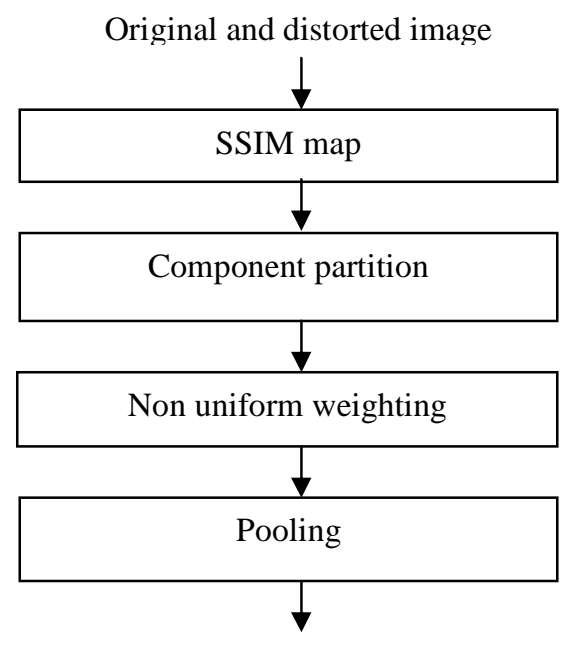

Final aualitv metric

Fig -4: Steps involved in content partitioned structural similarity index

\section{PERFORMANCE COMPARISON METHODS}

Each image quality assessment algorithm has its own advantages and disadvantages. The accuracy and efficiency of algorithms can be found by comparing the performance. For comparing the performance of image quality assessment algorithms, four performance comparison metrics are commonly used by most algorithms. These are Pearson linear correlation coefficient (PLCC), Kendall rank correlation coefficient (KRCC), Spearman rank correlation coefficient (SROCC) and Mean squared error (MSE) [8]. If the SROCC, KRCC, and PLCC values are higher and MSE value is lower, then that metric can be considered as a good one. The performance comparison can be done by comparing the mean opinion score (MOS) that is found by subjective rating and the objective score that is found by using the image quality measurement algorithms. Subjective scores are normally represented by Mean Opinion Score (MOS) or Differential mean opinion score (DMOS). For the implementation of these algorithms many databases can be used. These are publicly available online. This includes LIVE, CSIQ, TID2008 etc.

\section{CONCLUSIONS}

This survey shows various full reference image quality assessment algorithms such as SSIM, MAD, NPID, MSSSIM, FSIM, content portioned SSIM and practical image quality metric. All these IQA algorithms are pixel based or wavelet based. Some algorithms taking the structural information where as some are taking the energy features or other features for finding out the quality of the image. Each algorithm has its own merits and demerits. The complexity of some algorithms is more because of the large computational time and the use of complex mathematical equations. Based on the accuracy, time and other requirements we can select the algorithms.

Table -1: Summary of full reference IQA metrics

\begin{tabular}{|c|l|l|}
\hline $\begin{array}{c}\text { REFER- } \\
\text { ENCES }\end{array}$ & $\begin{array}{l}\text { METHODS } \\
\text { USED }\end{array}$ & \multicolumn{1}{c|}{ REMARKS } \\
\hline$[6]$ & $\begin{array}{l}\text { Structural } \\
\text { similarity } \\
\text { index } \\
\text { (SSIM) }\end{array}$ & $\begin{array}{l}\text { Can handle color images, } \\
\text { predict contrast change and } \\
\text { mean shift, Less effective when } \\
\text { used to rate badly blurred and } \\
\text { noisy images }\end{array}$ \\
\hline$[3]$ & $\begin{array}{l}\text { Most } \\
\text { apparent } \\
\text { distortion } \\
\text { (MAD) }\end{array}$ & $\begin{array}{l}\text { Improved prediction accuracy, } \\
\text { Perform well for both high } \\
\text { quality and low quality images, } \\
\text { Not suitable for Color Images }\end{array}$ \\
\hline$[9]$ & $\begin{array}{l}\text { Normalized } \\
\text { perceptual } \\
\text { information } \\
\text { distance } \\
\text { (NPID) }\end{array}$ & $\begin{array}{l}\text { Comparable prediction accuracy } \\
\text { with others, using the non } \\
\text { computable concept } \\
\text { kolmogorov } \\
\text { Computation time is higher }\end{array}$ \\
\hline$[7]$ & $\begin{array}{l}\text { Multi scale } \\
\text { structural } \\
\text { similarity for } \\
\text { image } \\
\text { quality } \\
\text { assessment } \\
\text { (MS-SSIM). }\end{array}$ & $\begin{array}{l}\text { A good approximation of the } \\
\text { perceived image quality. Giving } \\
\text { relative importance between } \\
\text { different scales, the prediction } \\
\text { capacity is higher, Fails in } \\
\text { measuring badly blurred } \\
\text { images. }\end{array}$ \\
\hline
\end{tabular}




\begin{tabular}{|c|l|l|}
\hline [5] & $\begin{array}{l}\text { A Feature } \\
\text { Similarity } \\
\text { Index for } \\
\text { Image } \\
\text { Quality } \\
\text { Assessment } \\
\text { (FSIM) }\end{array}$ & $\begin{array}{l}\text { Can apply to color images, } \\
\text { better predictability for quality, } \\
\text { robustness, covers nearly all } \\
\text { distortions, Relatively high } \\
\text { computation time }\end{array}$ \\
\hline$[1]$ & $\begin{array}{l}\text { Practical } \\
\text { image } \\
\text { quality } \\
\text { metric }\end{array}$ & $\begin{array}{l}\text { Easy to optimize, reliable for } \\
\text { non-uniform distortions, fast } \\
\text { and simple, distortion } \\
\text { calculation repeated several } \\
\text { times }\end{array}$ \\
\hline$[4]$ & $\begin{array}{l}\text { Content- } \\
\text { partitioned } \\
\text { structural } \\
\text { similarity } \\
\text { index }\end{array}$ & $\begin{array}{l}\text { Improved performance against } \\
\text { human subjectivity, Overhead } \\
\text { of setting weights, } \\
\text { Computation time is more }\end{array}$ \\
\hline
\end{tabular}

\section{ACKNOWLEDGEMENTS}

I would like to thank my project guide Mr. S. John Livingston for helping me in the field of image quality assessment.

\section{REFERENCES}

[1]. Zhang. F., Ma. Lin., Li. S. and Ngan. K. N. 2011, "Practical image quality metric applied to image coding", IEEE Trans. On Multimedia. 13(4), 615-624.

[2]. Wang. Z, Bovik. A. C., Mean squared error: love it or leave it? A new look at signal fidelity measures, IEEE Signal Process. Mag. 26 (1) (2009) 98-117.

[3]. Larson. E. C. and Chandler. D. M., "Most apparent distortion: full reference image quality assessment and the role of strategy", J. Electron. Imaging, 19, 011006:1-21, 2010.

[4]. Li. C. and Bovik. A. C., "Content partitioned structural similarity index for image quality assessment Signal processing: image communication 25, 517-526, 2011.

[5]. Zhang. Lin., Zhang. Lei., Mou. X. and Zhang. D., "FSIM: A feature similarity index for image quality assessment", IEEE Trans. Image Process. 20(8), 2378-2386, 2011.

[6]. Wang. Z., Bovik. A.C., Sheikh, H.R. and Simoncelli. E.P., "Image quality assessment: from error visibility to structural similarity”, IEEE Trans. Image Process. 13(4), 600-612, 2011. [7]. Wang. Z., Simoncelli. E.P. and Bovik. A.C., "Multi-scale structural similarity for image quality assessment", In: Proceedings of IEEE Asilomar Conference on Signals, Systems, and Computers (Pacific Grove, CA), pp. 1398-1402, 2003.

[8]. Wang. Z. and Li. Q., "Information content weighting for perceptual image quality assessment", IEEE Trans Image Process 20(5), 1185-1198, 2011

[9]. Nikvand. N and Wang. Z, "Image distortion analysis based on normalized perceptual information distance", SIViP (2013) 7:403-410.
[10]. Larson. E. C., Chandler. D.M.: Categorial image quality (CSIQ) database. (Online) Available http://vision.okstate.edu/csiq

[11]. Sheikh. H.R. and Bovik. A.C., "Image information and visual quality", IEEE Trans. Image Process. 15(2), 430-444, (2006)

[12]. Li. M., Chen. X., Li. X., Ma. B. and Vitanyi P. M. B., "The similarity metric", IEEE Trans Information Theory 50(12), 2004

[13]. Ponomarenko. N., Silvestri. F., Egiazarian. K., Carli. M., Astola. J. and Lukin. V. (2007) "On between-coefficient contrast masking of det basis functions", In: $3^{\text {rd }}$ International Workshop on Video Processing and functions. Scottsdale, Arizona, USA.

[14]. Wainwright. M.J., Simoncelli. E.P., "Scale mixtures of gaussians and statistics of natural images", In: Solla, S.A., Leen. T.K., Muller. K.R., (eds) Advances in neural information processing, vol. 12,pp. 855-861. MIT Press, Cambridge (2000)

\section{BIOGRAPHIES}

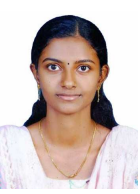

Alphy George received her B.Tech (Computer Science and Engineering) degree from SCMS school of Engineering and Technology affiliated by Mahatma Gandhi University in the year 2012. She is pursuing her M. Tech degree in Computer Science and Engineering at Karunya University.

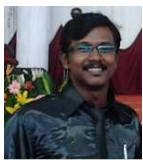

S. John Livingston received his M.E. degree from Karunya University. He is currently working as an Assistant Professor in Karunya University. 